\title{
Fractional Media-Based Modulation with Golden Angle Modulation
}

\author{
Ibrahim Yildirim*, Ertugrul Basar ${ }^{\dagger}$, and Ibrahim Altunbas* \\ *Faculty of Electrical and Electronics Enginnering, Istanbul Technical University, Sariyer 34469, Istanbul, Turkey \\ ${ }^{\dagger}$ CoreLab, Department of Electrical and Electronics Enginnering, Koç University, Sariyer 34450, Istanbul, Turkey \\ Email: yildirimib@itu.edu.tr, ebasar@ku.edu.tr, ibraltunbas@itu.edu.tr
}

\begin{abstract}
One of the main drawbacks in the use of reconfigurable antennas for index modulation (IM) is the need to obtain an integer power of two number of uncorrelated channel realizations. In this study, we present media-based modulation with golden angle modulation (GAM-MBM) scheme as a new solution to this fractional bits problem by using symmetrical structure of golden angle modulation (GAM). We present a comprehensive overview of the fractional bits problem in spatial modulation (SM) and media-based modulation (MBM) and we show that the proposed scheme can be considered as a candidate for the next generation technologies with its versatile structure, which can be easily integrated into various concepts. Simulation results also show that GAM-MBM exhibits significant improvements in error performance over the existing fractional bits solutions.
\end{abstract}

Index Terms-Channel modulation, index modulation, mediabased modulation, golden angle modulation, fractional bits.

\section{INTRODUCTION}

With the definition of the $5 \mathrm{G}$ vision, the demand for key requirements such as high data rate, low latency and low energy consumption from the perspective of next generation communication technologies, is ascending [1]. Miscellaneous candidate technologies have been explored and proposed to bring this visionary framework to reality. In order to fully understand these emerging technologies, comprehensive and multi-fold engineering paradigms are needed.

While it is possible to reach high data rates by increasing the spectral efficiency, it is confronted with high energy consumption and increased system complexity problems [2]. Owing to its low complexity and high energy efficiency, spatial modulation (SM), which is a sub-type of index modulation (IM) [3], [4], has attracted remarkable attention and has been extensively investigated in the past few years by researchers [5]-[8]. In SM, the conventional modulation symbol from the $Q$-QAM/PSK is sent through an active antenna which is selected among $N_{t}$ transmit antennas according to the index bits. Therefore, the spectral efficiency of SM systems is obtained as $\eta=\log _{2}\left(N_{t}\right)+\log _{2}(Q)$ bits per channel use.

The newest member of the IM family is media-based modulation (MBM), which is proposed for rich scattering wireless communication environments. In MBM, different channel fading realizations are obtained by changing the current distribution of the antenna by means of parasitic elements. In order to obtain high spectral efficiencies, these parasitic elements, called RF (radio frequency) mirrors, are placed near the transmit antenna [9], [10]. In this setup, $N_{r f}$ number of RF mirrors, controlled by digital switches, allow to obtain $M_{r f}=2^{N_{r f}}$ different channel states. Besides classical modulation methods such as $Q$-PSK and $Q$-QAM, different channel states are indexed and used as a new dimension to convey additional information bits. In this way, the spectral efficiency of MBM systems is expressed as $\eta=N_{r f}+\log _{2}(Q)$ bits per channel use. Thus, this concept is expected to achieve high data rates without deteriorating error performance using only one RF chain [11]-[14]. Besides, one of the limitation of MBM is that the number of different channel states must be an integer power of two. Both SM and MBM systems suffer from the fractional bits problem and index part of SM and MBM are expected to be an integer to overcome this issue.

In this work, with the motivation of exploiting fractional bit encoding, we propose a comprehensive solution that is applicable for both MBM and SM. With the aim of reaching an inclusive solution, the problem should be addressed from the perspective of both index and modulation bits, not only in terms of index bits. PSK/QAM modulation types play a restrictive role here as well as index modulation techniques. In the $Q$-ary PSK/QAM, if $Q$ is not equal to an integer power of two, particularly QAM method loses most of its advantages and the constellation space should be redesigned since constellation symmetry is no longer valid. Non-uniform constellations (NUC) have been proposed by authors in [15], in order to avoid orthogonal grid constraints of QAM and to provide higher shaping gain. Similar to the NUC designs, in [16], the author proposed the golden angle modulation (GAM), which did not exhibit asymptotic loss, as an alternative to QAM. When the number of constellation points $Q$ goes to infinity, the mutual information has been shown to be close to Shannon capacity. This type of modulation, inspired by plants such as leaf, seed, which have packing arrangements in the form of spiral phyllotaxis in nature, shows the same symmetrical distribution as seen in Fig. 1, regardless of its size. Although two different GAM schemes were proposed as disc-GAM and geometric-bell-GAM, disc-GAM is used in this article owing to its simple structure. As can be seen from Fig. 2, disc-GAM offers a flexible solution to the fractional bits problem, since it has a symmetrical distribution even when the constellation size is not an integer power of two.

In this context, the aim of this paper is to design a novel 


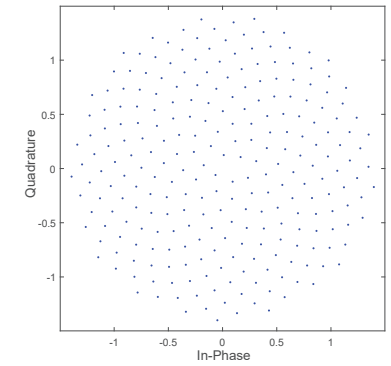

(a) Signal constellation of 256-disc- (b) Signal constellation of 2048-discGAM.

Fig. 1. The constellation points of disc-GAM-MBM for non-fractional case

scheme, which combines GAM and MBM, to break the limitation of fractional bits in IM while pursuing the high spectral efficiency and enhancing error performance of IM. Performance results show that the proposed schemes achieve satisfactory error performance for different number of antennas and RF mirrors while only one RF chain used at the transmitter side.

The remainder of this paper is organized as follows. Section II briefly describes the concept of fractional bits problem and disc-GAM constellation. In Section III, the proposed schemes are presented and discussed in detail. After pointing out its generalizations for different number of antennas and RF mirrors, an analysis of the pairwise error probability (PEP) performance is given. Finally, Monte Carlo simulation results are reported in Section IV, and the paper is concluded in Section V.

\section{BACKGROUND}

\section{A. Fractional Bits Problem}

One of the shortcomings of SM is the restriction of the number of transmit antennas to an integer power of two. This means that the index part of incoming bits $\left(\log _{2}\left(N_{T}\right)\right)$ must be an integer. By virtue of breaking this restriction, the fractional bit encoded spatial modulation (FBE-SM) based on the modulus conversion was proposed in [17]. However, FBE-SM does not offer a flexible design when using different number of transmit antennas and causes error propagation that worsens error performance. Furthermore, generalized SM (GSM) technique solves this problem by activating more than one antenna at the same time in SM, however, it cannot be considered as a solution to this problem in MBM since an antenna cannot be set to generate multiple channel states at the same time [18]. On the other hand, in the bit padding method, which was proposed as an alternative to the FBE, the padding bits must be removed to recover the data [19]. Any errors in the depadding process will cause the detector to detect the data incorrectly. In [20], 3-D constellation design for jointly mapped spatial modulation (JM-SM), in which antenna indices and PSK/QAM symbols are jointly modulated, was proposed. In JM-SM, a set of $2^{\eta}$ possible transmission vectors, where $\eta$ is the spectral efficiency, is created by selecting a variable constellation symbol set for each transmit

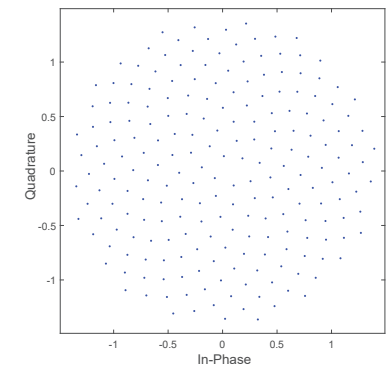

(a) Signal constellation of 213-discGAM.

Fig. 2. The constellation points of disc-GAM-MBM for fractional case.

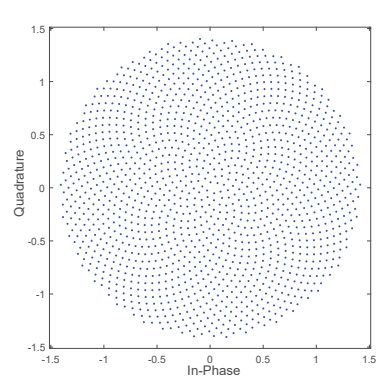

(b) Signal constellation of 1445-discGAM. antenna. Using optimization techniques, a variable number of symbols are selected from $Q$-ary constellation points for each antenna. This optimization-based selection process leads to inefficient usage of $Q$-ary modulation symbols and an increase in the transmitter's complexity. In order to bypass the number of transmit antenna limitation of SM, the authors proposed a single RF-aided SM scheme for an arbitrary number of transmit antennas (SM-ATA) [21]. The SM-ATA scheme, in which the $Q$-ary modulation symbols are rotated to adjust the total number of possible transmission vectors to be an integer power of two, imposes deteriorated error performance when the degree of modulation $Q$ increases.

Similar to SM, the problem of the fractional index bits is encountered in MBM systems. In MBM, the available $N_{r f}$ RF mirrors are expected to produce $2^{N_{r f}}$ number of different radiation patterns, however, in practice some of these patterns can be highly correlated for particular cases. Similarity of the channel states causes incorrect decoding of index bits at the receiver. In order to circumvent this imperfection, data transmission can be done using uncorrelated channel states among the $2^{N_{r f}}$ different states. As the number of channel states used is not an integer power of two, the fractional bit problem can more generally occur in MBM. However, when the schemes proposed for SM are adapted to MBM, an effective solution cannot be provided. In the light of above, there is a need of a method that works in both MBM and SM realizations, requires a single RF chain at the transmitter side, does not require optimization, and does not waste energy using the whole of the modulation space.

\section{B. Disc-GAM}

General expression of the $n$th constellation point of GAM with equal probability can be shown as

$$
s_{n}=r_{n} e^{i 2 \pi \varphi n}, n \in\{1,2, \ldots, Q\},
$$

where $r_{n}$ is the amplitude of $n$th constellation symbol, $\varphi$ represents the golden angle $(\varphi=1-(\sqrt{5}-1) / 2)$ and $Q$ is the size of the constellation [16]. In this work, disc-GAM, which has a disc-shaped constellation distribution, is used. For the $n$th symbol of the constellation, the radius of disc-GAM symbol is expressed as

$$
r_{n}=c \sqrt{n}, n \in\{1,2, \ldots, Q\}
$$



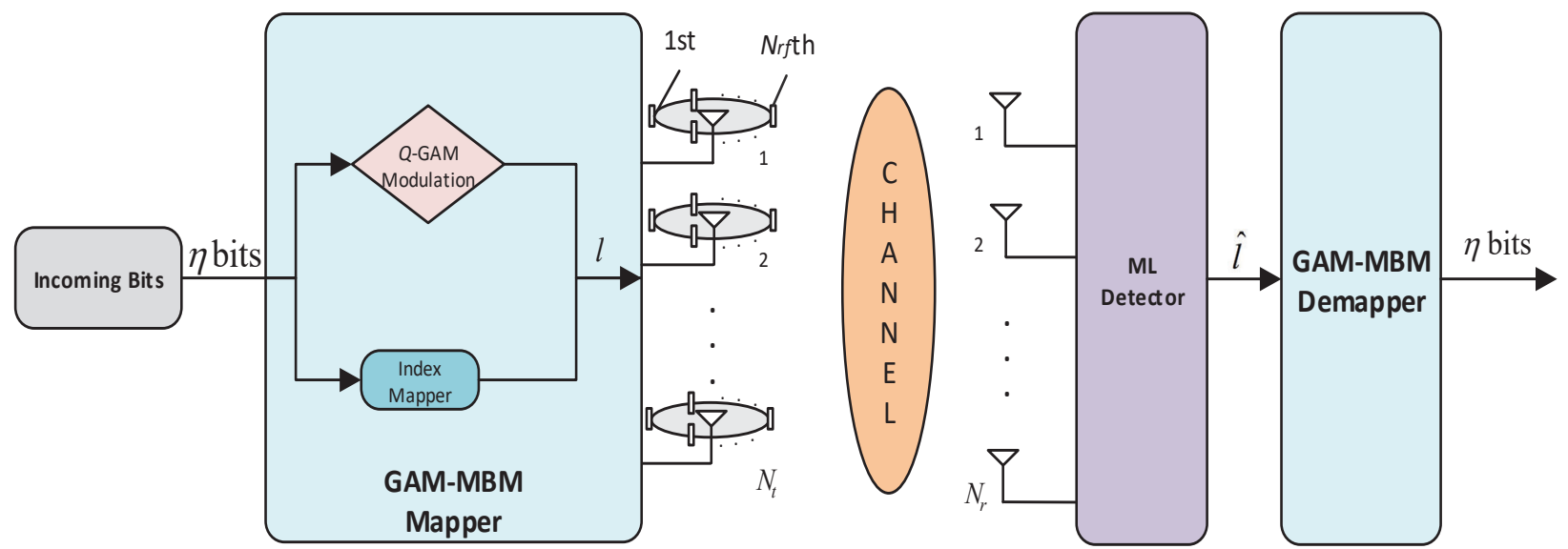

Fig. 3. Block diagram of the GAM-MBM schemes for an $N_{r} \times N_{t}$ MIMO system with $N_{r f}$ RF mirrors at each transmit antenna.

$$
c \triangleq \sqrt{\frac{2 P}{Q+1}},
$$

where $P$ is the average power constraint. In Fig.1, disc-GAM signal constellations for $Q=256$ and $Q=2048$ are depicted. The disc-GAM scheme maintains the properties it offers for any degree of constellation whether or not $Q$ is an integer power of two. As shown in Fig. 2, using each point of signal constellation effectively, a flexible structure is obtained, even if the constellation size is not an integer power of two. GAM schemes were proposed for applications with limited resources in the transmitter in terms of power, energy, and complexity [16]. In systems, where high decoding complexity can be ignored, GAM provides significant advantages.

\section{Media-Based Modulation with Golden Angle MODULATION}

A multiple-input multiple-output (MIMO) system, in which modulated symbols are sent from $N_{t}$ transmit antennas configured by $N_{r f}$ RF mirrors to $N_{r}$ receive antennas, is considered, as shown in Fig. 3. In the proposed MBM with golden angle modulation (GAM-MBM) scheme, the set of possible transmission vectors are created by configuring the system design through the targeted transmission rate. For a system with spectral efficiency $\eta$ bits per channel use, $2^{\eta}$ transmission vectors are formed to be transmitted from $N_{t} m_{r f}$ different channel realizations, where $m_{r f}$ represents the number of different uncorrelated channel states at each transmit antenna $\left(m_{r f} \leq 2^{N_{r f}}\right)$. Incoming bits directly determine the transmission vector $\mathbf{x}_{l}, l=1,2, \ldots, 2^{\eta}$, which are of the form:

$\mathbf{x}_{l} \in\left\{\left[\begin{array}{c}s_{1}^{\prime} \\ 0 \\ \vdots \\ 0\end{array}\right], \ldots,\left[\begin{array}{c}s_{Q_{1}}^{\prime} \\ 0 \\ \vdots \\ 0\end{array}\right],\left[\begin{array}{c}0 \\ s_{1}^{\prime} \\ \vdots \\ 0\end{array}\right], \ldots,\left[\begin{array}{c}0 \\ s_{Q_{1}}^{\prime} \\ \vdots \\ 0\end{array}\right], \ldots,\left[\begin{array}{c}0 \\ \vdots \\ 0 \\ s_{1}^{\prime \prime}\end{array}\right], \ldots,\left[\begin{array}{c}0 \\ \vdots \\ 0 \\ s_{Q_{2}}^{\prime \prime}\end{array}\right]\right\}$

where $s_{i}^{\prime}\left(i=1,2, \ldots, Q_{1}\right)$ and $s_{j}^{\prime \prime}\left(j=1,2, \ldots, Q_{2}\right)$ represent the sub-signal constellation symbols sent from the first $n_{1}$ and the remaining $n_{2}$ channel realizations $\left(n_{1}+n_{2}=N_{t} m_{r f}\right)$, respectively. $n_{1}$ and $n_{2}$ are defined as follows:

$$
\begin{aligned}
& n_{1}=2^{\eta}-\left\lfloor\frac{2^{\eta}}{N_{t} m_{r f}}\right\rfloor N_{t} m_{r f} \\
& n_{2}=N_{t} m_{r f}-n_{1} .
\end{aligned}
$$

TABLE I

MAPPING RULE FOR $N_{t}=1, N_{r f}=2, m_{r f}=3$ AND $\eta=4$ BPCU.

\begin{tabular}{ccc}
\hline \hline Incoming Bits & Vector Index & Transmission Vector \\
\hline & & {$\left[s_{1}^{\prime}, 0,0\right]^{T}$} \\
0000 & 1 & {$\left[s_{2}^{\prime}, 0,0\right]^{T}$} \\
0001 & 2 & {$\left[s_{3}^{\prime}, 0,0\right]^{T}$} \\
0010 & 3 & {$\left[s_{4}^{\prime}, 0,0\right]^{T}$} \\
0011 & 4 & {$\left[s_{5}^{\prime}, 0,0\right]^{T}$} \\
0100 & 5 & {$\left[s_{6}^{\prime}, 0,0\right]^{T}$} \\
0101 & 6 & {$\left[0, s_{1}^{\prime \prime}, 0\right]^{T}$} \\
0110 & 7 & {$\left[0, s_{2}^{\prime \prime}, 0\right]^{T}$} \\
0111 & 8 & {$\left[0, s_{3}^{\prime \prime}, 0\right]^{T}$} \\
1000 & 9 & {$\left[0, s_{4}^{\prime \prime}, 0\right]^{T}$} \\
1001 & 10 & {$\left[0, s_{5}^{\prime \prime}, 0\right]^{T}$} \\
1010 & 11 & {$\left[0,0, s_{1}^{\prime \prime}\right]^{T}$} \\
1011 & 12 & {$\left[0,0, s_{2}^{\prime \prime}\right]^{T}$} \\
1100 & 13 & {$\left[0,0, s_{3}^{\prime \prime}\right]^{T}$} \\
1101 & 14 & {$\left[0,0, s_{4}^{\prime \prime}\right]^{T}$} \\
1110 & 15 & {$\left[0,0, s_{5}^{\prime \prime}\right]^{T}$} \\
1111 & 16 &
\end{tabular}

On the other hand, the dimensions of two sub-signal constellations are denoted by $Q_{1}$ and $Q_{2}$ are expressed as

$$
\begin{aligned}
Q_{1} & =\left\lfloor\frac{2^{\eta}}{N_{t} m_{r f}}\right\rfloor N_{t} m_{r f}+1 \\
Q_{2} & =\left\lfloor\frac{2^{\eta}}{N_{t} m_{r f}}\right\rfloor N_{t} m_{r f} .
\end{aligned}
$$




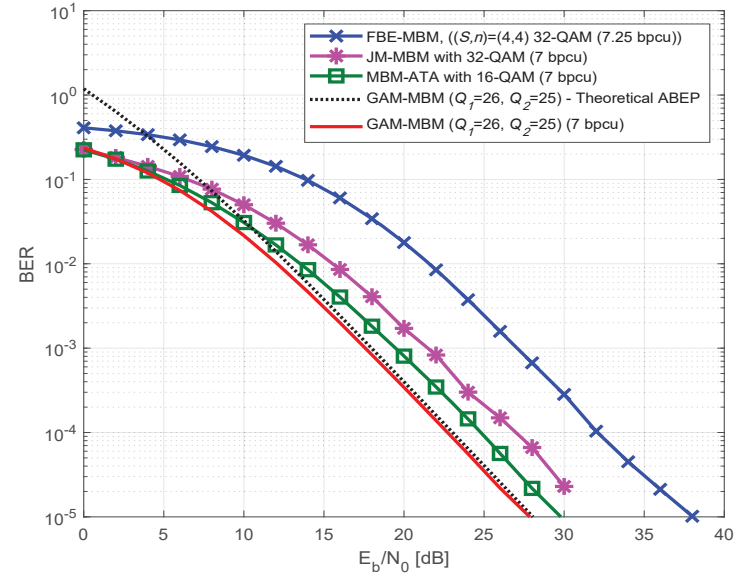

Fig. 4. BER comparison of GAM-MBM versus reference systems for $\eta \cong 7$ bpcu $\left(N_{t}=1, N_{r}=2, N_{r f}=2, m_{r f}=3\right)$.

Following this procedure, we give an example for the GAMMBM. Assume that the system has the following parameters: $N_{t}=1, N_{r f}=2, m_{r f}=3$ and $\eta=4$ bpcu. Using (5), the different channel realizations of the first and second constellation group are calculated as $n_{1}=1$ and $n_{2}=2$, respectively. This means that $n_{1}=1$ and $n_{2}=2$ channel states from available $N_{t} m_{r f}=3$ channel realizations are used to transmit $Q_{1}$-ary and $Q_{2}$-ary GAM symbols, where their sizes are determined as $Q_{1}=6$ and $Q_{2}=5$ via (6), respectively. The mapping rule of this example is shown Table I.

The received signal $\mathbf{y} \in \mathbb{C}^{N_{r} \times 1}$ of the GAM-MBM at the output of the channel is given by

$$
\mathbf{y}=\mathbf{H} \mathbf{x}_{l}+\mathbf{w}
$$

where $\mathbf{H} \in \mathbb{C}^{N_{r} \times N_{t} m_{r f}}$ and $\mathbf{w} \in \mathbb{C}^{N_{r} \times 1}$ denote the extended channel matrix and the Gaussian noise vector, respectively. It is assumed that $\mathbf{H}$ and $\mathbf{w}$ distributed as $C N(0,1)$ and $C N\left(0, N_{0}\right)$, respectively.

At the receiver, the index of the transmitted vector index is determined using the maximum-likelihood (ML) principle, as follows,

$$
\hat{l}=\arg \min _{l}\left\|\mathbf{y}-\mathbf{H} \mathbf{x}_{l}\right\|^{2}
$$

where $\|$.$\| denotes the Euclidean norm.$

The performance of the GAM-MBM scheme is derived using the common union bounding technique. The average bit error probability (ABEP) of the GAM-MBM is bounded with

$$
P_{b} \leq \frac{1}{\eta 2^{\eta}} \sum_{\mathbf{x}_{l}} \sum_{\hat{\mathbf{x}}_{l}} P\left(\mathbf{x} \rightarrow \hat{\mathbf{x}}_{l}\right) e\left(\mathbf{x}, \hat{\mathbf{x}}_{l}\right)
$$

where $e\left(\mathbf{x}, \hat{\mathbf{x}}_{l}\right)$ is the number of different bits in which $\mathbf{x}_{l}$ is detected as $\hat{\mathbf{x}}_{l}$ and $P\left(\mathbf{x} \rightarrow \hat{\mathbf{x}}_{l}\right)$ is the unconditional pairwise error probability (PEP), which is given by [22]

$$
P\left(\mathbf{x}_{l} \rightarrow \hat{\mathbf{x}}_{l}\right)=\frac{1}{\pi} \int_{0}^{\frac{\pi}{2}}\left(\frac{\sin ^{2} \theta}{\sin ^{2} \theta+\frac{\left\|\mathbf{x}_{l}-\hat{\mathbf{x}}_{l}\right\|^{2}}{4 N_{0}}}\right)^{N_{r}} d \theta .
$$

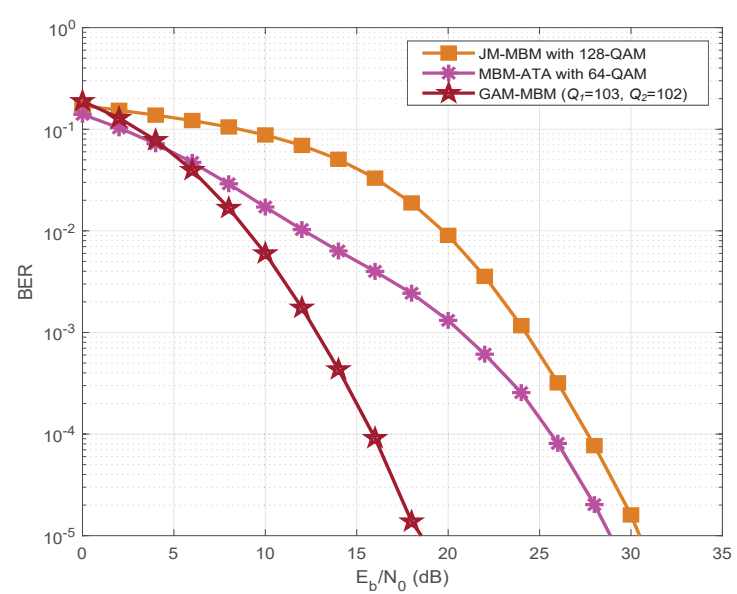

Fig. 5. BER comparison of GAM-MBM versus reference systems for $\eta=11$ bpcu $\left(N_{t}=4, N_{r}=4, N_{r f}=2, m_{r f}=3\right)$.

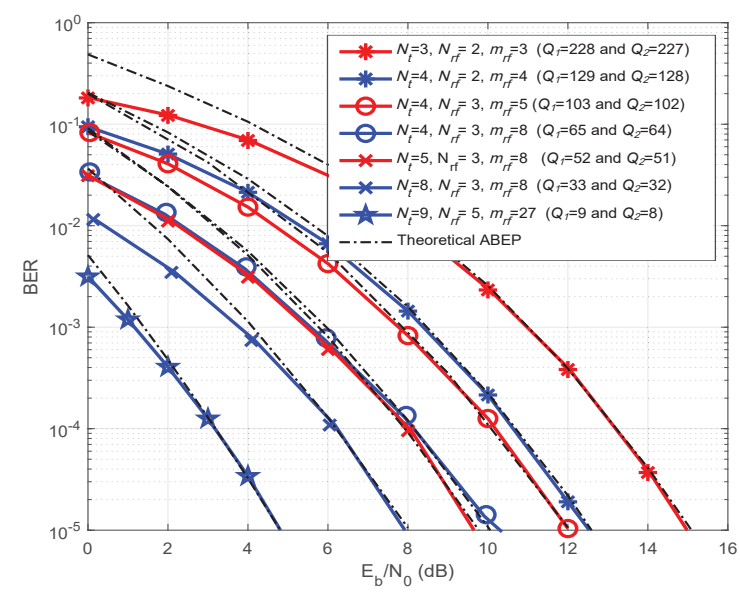

Fig. 6. BER performance and the ABEP curves of the GAM-MBM for the varying $N_{t}$ and $N_{r f}\left(N_{r}=8, \eta=11 \mathrm{bpcu}\right)$.

\section{NUMERICAL StUdies}

In the following, numerical results of the proposed GAMMBM are presented. Numerical results are obtained for different system configurations considering Rayleigh flat fading wireless channel. By Monte Carlo simulations, BER results are obtained and compared to analytical ABEP results with respect to signal-to-noise ratio (SNR). SNR is defined as $E_{b} / N_{0}$ where $E_{b}$ is the average transmitted energy per bit.

First of all, the performances of the methods proposed for the fractional bits case of SM, such as FBE-SM [17], JMSM [20], and SM-ATA [21], are analyzed in conjunction with MBM to match with GAM-MBM. In order to make a fair comparison, non-optimized versions of the existing methods are used in simulations.

Fig. 4 demonstrates the error performance curve of the proposed scheme for $\eta=7 \mathrm{bpcu}$ and its comparison with the reference systems for the same or very close data rates. The MBM-adapted versions of FBE-SM, JM-SM, and SM-ATA are 
named as FBE-MBM, JM-MBM and MBM-ATA, respectively. As seen from Fig. 4, GAM-MBM is superior to these reference schemes, for two receive antennas, single transmit antenna and two RF mirrors. Under these single-input multiple-output (SIMO) configurations, $N_{t} m_{r f}=3$ uncorrelated channel states could be produced instead of $N_{t} 2^{N_{r f}}=4$ different channel states and fractional methods have been used to compensate this effect.

In Fig. 5, we provide BER performance of the GAMMBM for the MIMO case corresponding to the SM-MBM combination. When both the number of transmit antennas and the number of channel states controlled by the RF mirrors are not an integer power of two, the fractional bits problem is solved and system performance is enhanced. It is obviously seen from the Fig. 5 that the superiority of the proposed GAM-MBM scheme becomes clear as the number of antennas increases.

Finally, the BER performance of the GAM-MBM, which uses a single RF chain, is illustrated in Fig. 6 considering varying number of transmit antennas and the number of channel states produced in each antenna. Fig. 6 also illustrates the conventional (non-fractional) SM-MBM combination, in which the number of antennas and channel states are equal to an integer power of two $\left(N_{t}=4, N_{r f}=2, m_{r f}=4\right.$ and $N_{t}=8$, $\left.N_{r f}=3, m_{r f}=8\right)$. It is observed that the error performance is improved when a higher number of channels are used for indexing to convey bits at the same spectral efficiency.

\section{Conclusions}

In this work, we have proposed the versatile GAM-MBM concept to break the limitation of fractional bits. The GAM has been examined from the IM perspective and a flexible transmitter design, which works in both MBM and SM schemes, requires a single RF chain at the transmitter side, neither requires optimization nor wastes energy using every part of the signal constellation, has been introduced. While the sophisticated GAM-MBM scheme provides a comprehensive solution to the fractional bits problem, it has made significant improvements in error performance as seen from the numerical examples. Considering all these outcomes, we conclude that the GAM-MBM scheme has the potential to be a candidate for next-generation communication technologies. Furthermore, the design of the GAM-MBM schemes with probabilistic optimization is planned as our future endeavor.

\section{ACKNOWLEDGMENT}

This work was supported by the Scientific and Technological Research Council of Turkey (TUBITAK) under Grant 117 E869 and Research Fund of the Istanbul Technical University (Project number: MYL-2019-41967).

The work of E. Basar was supported by the Turkish Academy of Sciences Outstanding Young Scientist Award Programme (TUBA-GEBIP).

\section{REFERENCES}

[1] S. Chen and J. Zhao, "The requirements, challenges, and technologies for $5 \mathrm{G}$ of terrestrial mobile telecommunication," IEEE Commun. Mag., vol. 52, no. 5, pp. 36-43, May 2014.

[2] M. Di Renzo, H. Haas, A. Ghrayeb, S. Sugiura, and L. Hanzo, "Spatial modulation for generalized MIMO: Challenges, opportunities, and implementation," Proc. of the IEEE, vol. 102, no. 1, pp. 56-103, Jan. 2014.

[3] E. Basar, "Index modulation techniques for $5 \mathrm{G}$ wireless networks," IEEE Commun. Mag., vol. 54, no. 7, pp. 168-175, July 2016.

[4] E. Basar, M. Wen, R. Mesleh, M. D. Renzo, Y. Xiao, and H. Haas, "Index modulation techniques for next-generation wireless networks," IEEE Access, vol. 5, no. 1, pp. 16693-16746, Sep. 2017.

[5] R. Mesleh, H. Haas, S. Sinanovic, C. W. Ahn, and S. Yun, "Spatial modulation," IEEE Trans. Veh. Technol., vol. 57, no. 4, pp. 2228-2241, July 2008.

[6] R. Mesleh, S. Ikki, and H. Aggoune, "Quadrature spatial modulation," IEEE Trans. Veh. Technol., vol. 64, no. 6, pp. 2738-2742, June 2015.

[7] J. Jeganathan, A. Ghrayeb, and L. Szczecinski, "Generalized space shift keying modulation for MIMO channels," in 2008 IEEE Int. Symp. Personal, Indoor, Mobile Radio Commun. (PIMRC), Cannes, France, Sep. 2008, pp. 1-5.

[8] E. Basar, U. Aygolu, E. Panayirci, and H. V. Poor, "Space-time block coded spatial modulation," IEEE Trans. Commun., vol. 59, no. 3, pp. 823-832, Mar. 2011.

[9] A. K. Khandani, "Media-based modulation: Converting static Rayleigh fading to AWGN," in IEEE Int. Symp. Inf. Theory, Honolulu, HI, USA, Jun. 2014, pp. 1549-1553.

[10] E. Seifi, M. Atamanesh, and A. K. Khandani, "Media-based MIMO A new frontier in wireless communication," Oct. 2015. [Online]. Available: arxiv.org/abs/1507.07516

[11] Y. Naresh and A. Chockalingam, "On media-based modulation using RF mirrors,” IEEE Trans. Veh. Technol., vol. 66, no. 6, pp. 4967-4983, Oct. 2016.

[12] E. Basar and I. Altunbas, "Space-time channel modulation," IEEE Trans. Veh. Technol., vol. 66, no. 8, pp. 7609-7614, Aug. 2017.

[13] I. Yildirim, E. Basar, and I. Altunbas, "Quadrature channel modulation," IEEE Wireless Commun. Lett., vol. 6, no. 6, pp. 790-793, Aug. 2017.

[14] Z. Bouida, H. El-Sallabi, A. Ghrayeb, and K. A. Qaraqe, "Reconfigurable antenna-based space-shift keying (SSK) for MIMO Rician channels," IEEE Trans. Wireless Commun., vol. 15, no. 1, pp. 446-457, Jan. 2016.

[15] N. S. Loghin, J. Zollner, B. Mouchouche, D. Ansorregui, J. Kim, and S. I. Park, "Non-uniform constellations for ATSC 3.0," IEEE Trans. Broadcast., vol. 62, no. 1, pp. 197-203, Mar. 2016.

[16] P. Larsson, "Golden angle modulation," IEEE Wireless Commun. Lett., vol. 7, no. 1, pp. 98-101, Feb. 2018.

[17] N. Serafimovski, M. Di Renzo, S. Sinanovic, R. Mesleh, and H. Haas, "Fractional bit encoded spatial modulation (FBE-SM)," IEEE Commun. Lett., vol. 14, no. 5, pp. 429-431, May 2010.

[18] A. Younis, N. Serafimovski, R. Mesleh, and H. Haas, "Generalised spatial modulation," in 2010 Conf. Record of the 44th Asilomar Conf. on Signals, Syst. and Comput., Pacific Grove, CA, USA, Nov. 2010, pp. 1498-1502.

[19] Y. Yang and S. Aissa, "Bit-padding information guided channel hopping," IEEE Commun. Lett., vol. 15, no. 2, pp. 163-165, Feb. 2011.

[20] S. Guo, H. Zhang, S. Jin, and P. Zhang, "Spatial modulation via 3-D mapping," IEEE Commun. Lett., vol. 20, no. 6, pp. 1096-1099, Jun. 2016.

[21] L. Xiao, Y. Xiao, L. You, P. Yang, S. Li, and L. Hanzo, "Single-RF and twin-RF spatial modulation for an arbitrary number of transmit antennas," IEEE Trans. Veh. Technol., vol. 67, no. 7, pp. 6311-6324, Dec. 2017.

[22] M. Simon and M. S. Alaouni, Digital Communications over Fading Channels. New York: John Wiley \& Sons, 2005. 